\title{
Human vs. Automatic Measurement of Biometric Sample Quality
}

Andy Adler, Tanya Dembinsky

University of Ottawa 


\section{Background}

- Measures of biometric quality are notoriously difficult

- Typically, we have considered (implicitly or explicitly) humans to be the correct judge of quality

- We wanted to understand the relationship between human quality measures and those from machines 


\section{Experiments}

\begin{tabular}{|l|l|l|}
\hline & \multicolumn{1}{|c|}{$\begin{array}{c}\text { Face } \\
\text { Mugshot DB }\end{array}$} & \multicolumn{1}{|c|}{$\begin{array}{c}\text { Iris } \\
\text { Our DB }\end{array}$} \\
\hline Human Quality & 8 subjects & 8 subjects \\
\hline $\begin{array}{l}\text { Biometric } \\
\text { Quality }\end{array}$ & 6 algorithms & 1 algorithm \\
\hline $\begin{array}{l}\text { Image Quality } \\
\text { Measures }\end{array}$ & IQM $^{1}$ & IQM $^{1}$ \\
\hline
\end{tabular}

$1_{\text {www.mitre.org/tech/mtf/ }}$ 


\section{Human Quality Evaluation}

Image Quality Assessment - Microsoft Internet Explorer

$-\square x$

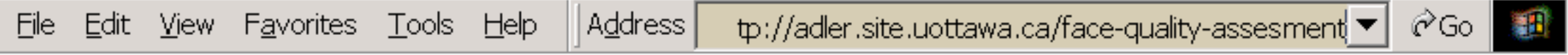

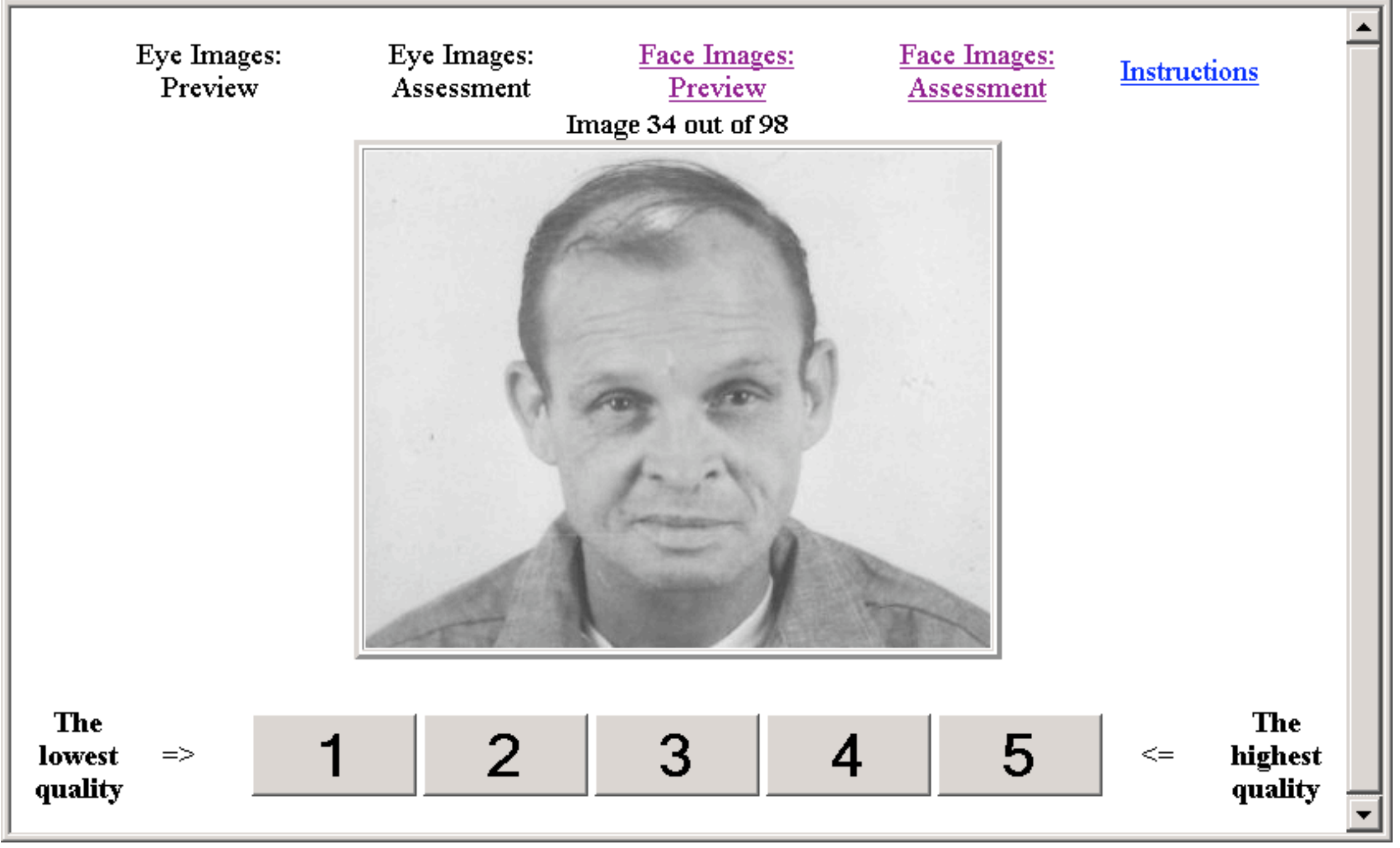




\section{Issues in Human Evaluations}

- Scale differences

- Analysis cannot compare raw values

- Training Effect

- Users were allowed to familiarize with database

- What is evaluated?

- Instructions were: "assess biometric image quality" 


\section{Quality from Match scores}

Model: MS from genuine comparisons is

due to image qualities

Except:

- Identical comparisons

- Different pose / age / etc.

$$
M S_{i, j}=Q_{i} Q_{j} \quad \begin{aligned}
& 0<M S<1 \\
& 0<Q<1
\end{aligned}
$$




\section{Quality from Match Scores}

Match Score

$$
\log M S_{i, j}=\log Q_{i}+\log Q_{j}
$$

Table

$\left.\begin{array}{|c|c|c|c|c|}\hline & 1 & 2 & 3 & 4 \\ \hline 1 & 1.0 & .9 & .8 & \\ \hline 2 & & 1.0 & .7 \\ \hline 3 & & & 1.0 & \\ \hline 4 & & & & 1.0\end{array}\right]\left[\begin{array}{cccc}1 & 1 & 0 & 0 \\ 1 & 0 & 1 & 0 \\ 0 & 1 & 1 & 0 \\ \vdots & & & \vdots\end{array}\right]\left[\begin{array}{l}\log Q_{1} \\ \log Q_{2} \\ \log Q_{3} \\ \log Q_{4}\end{array}\right]=\left[\begin{array}{c}\log .9 \\ \log .8 \\ \log .7 \\ \vdots\end{array}\right]$




\section{Comparisons}

- Are humans consistent with each other?

- Are algorithms consistent with each other?

- Are humans consistent with algorithms, or other quality measures? 


\section{Are humans consistent?}

Face

- Yes $(p<.001)$

- Average correlation coefficient $r=.613$

Iris

- Yes $(p<.001)$

- Average correlation coefficient $r=.723$ 


\section{Are algorithms consistent?}

Face

- Yes $(p<.001)$

- Average correlation coefficient $r=.534$

- Highest correlations not between different versions of same vendors SW

Iris

- Could not analyse (only one alg.) 


\section{Humans vs. algorithms}

Face

Iris

\begin{tabular}{|c|c|c|c|}
\hline & $\begin{array}{c}\text { Mean } \\
\text { Human }\end{array}$ & $\begin{array}{c}\text { Mean } \\
\text { FR Alg }\end{array}$ & IQM \\
\hline $\begin{array}{c}\text { Mean } \\
\text { Human }\end{array}$ & & .234 & .159 \\
\hline $\begin{array}{c}\text { Mean } \\
\text { Alg }\end{array}$ & .175 & & .003 \\
\hline IQM & .458 & -0.036 \\
\hline
\end{tabular}


- Best Faces

Worst Faces $\rightarrow$

Human Selections
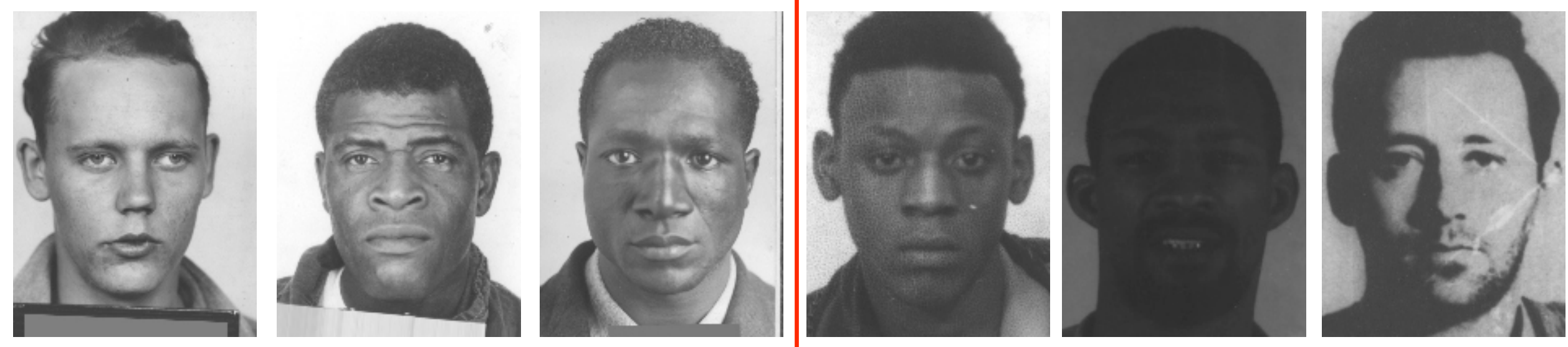

Algorithm Selections
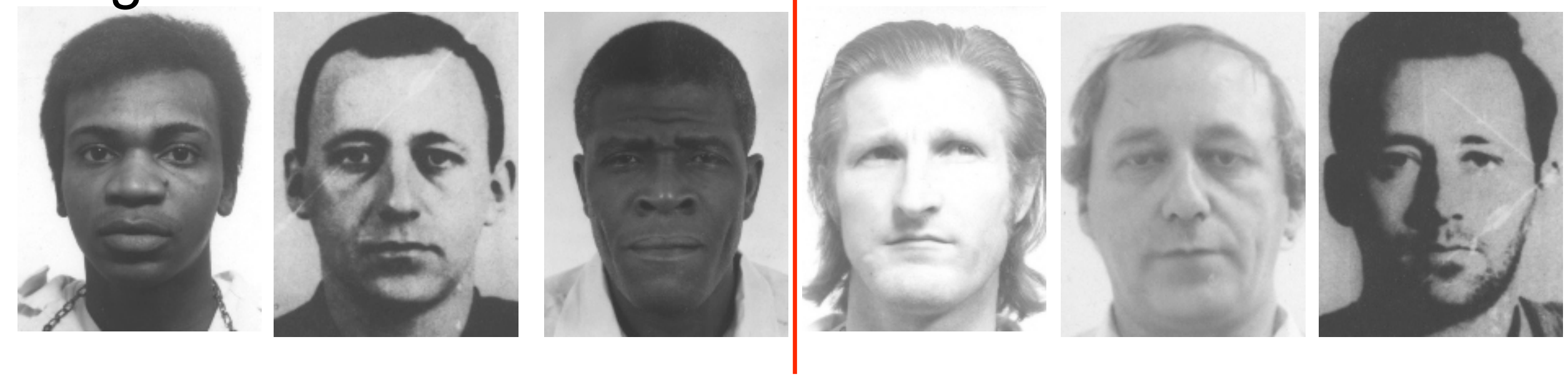
4 Best Irises

Human Selections

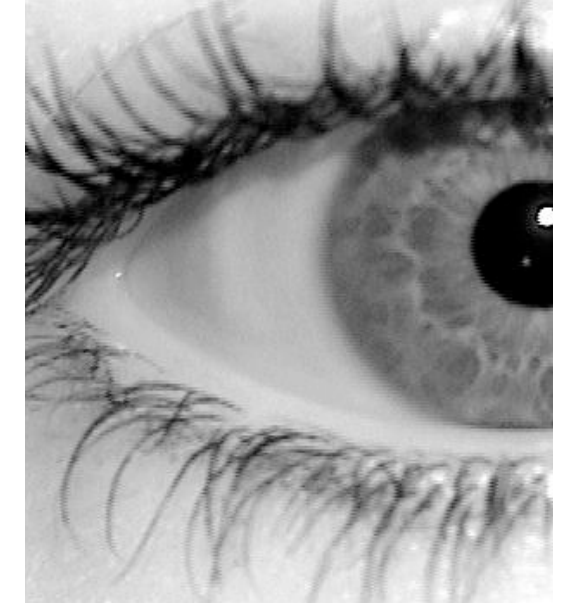

Algorithm Selections

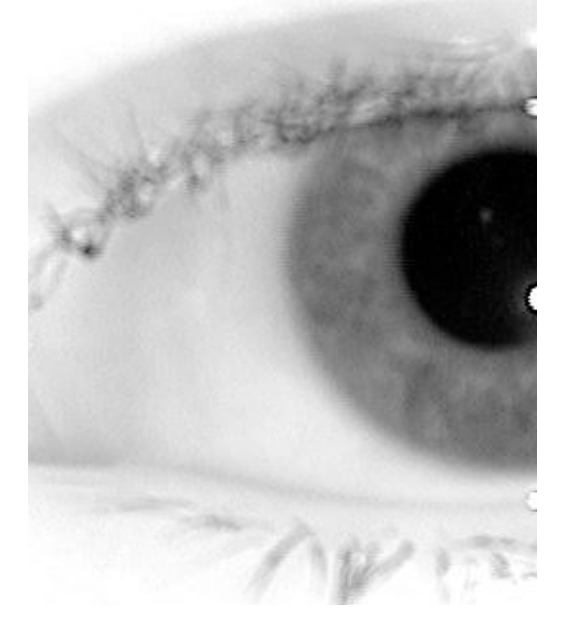

\section{Worst Irises $\rightarrow$}
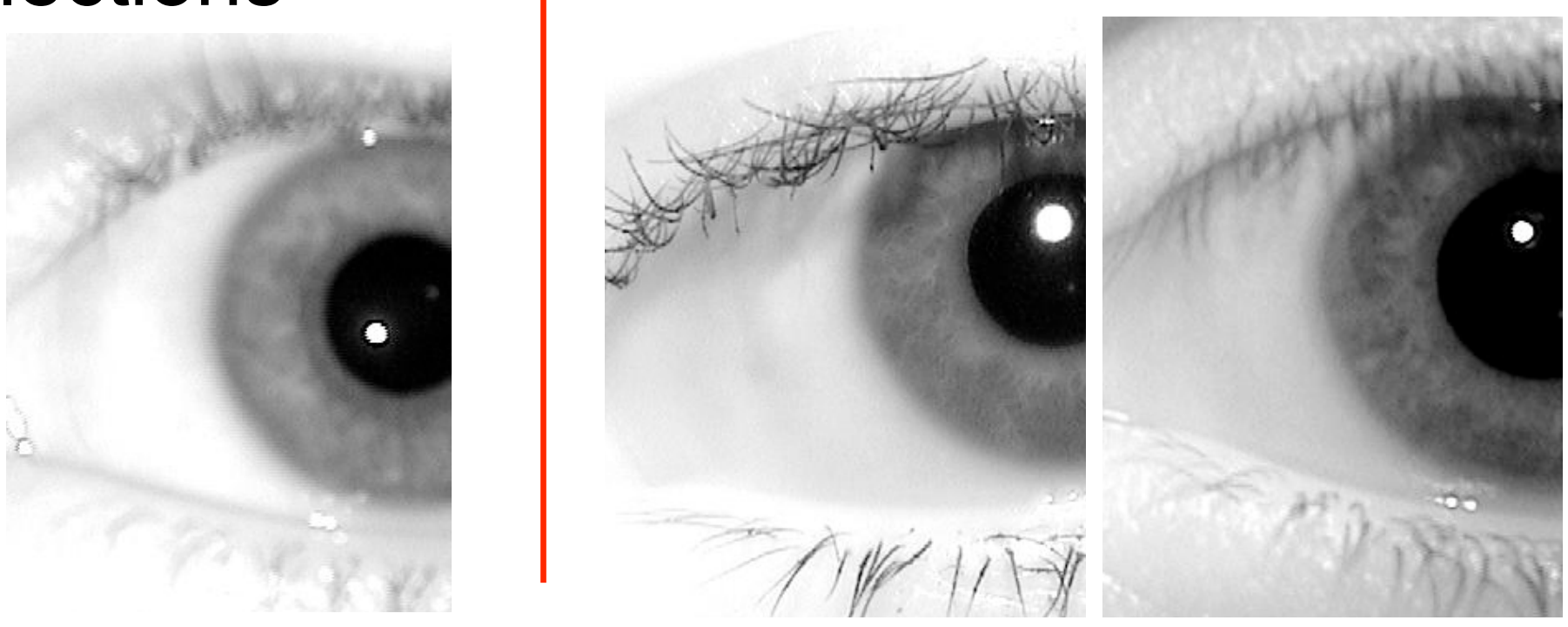


\section{Discussion}

- Work done on Face / Iris.

- Fingerprints are different because there are fingerprint experts

- Humans are consistent

- Algorithms are consistent

- But, humans are not consistent with algorithms 


\section{What does this mean?}

- Naïve ideas about quality measures may not be relevant to algorithms

- Some countries are vetting submitted passport photos for Face Rec

- How useful is this really? 


\section{Comment: Quality}

- Quality is a value laden term

- Can we tell users this?

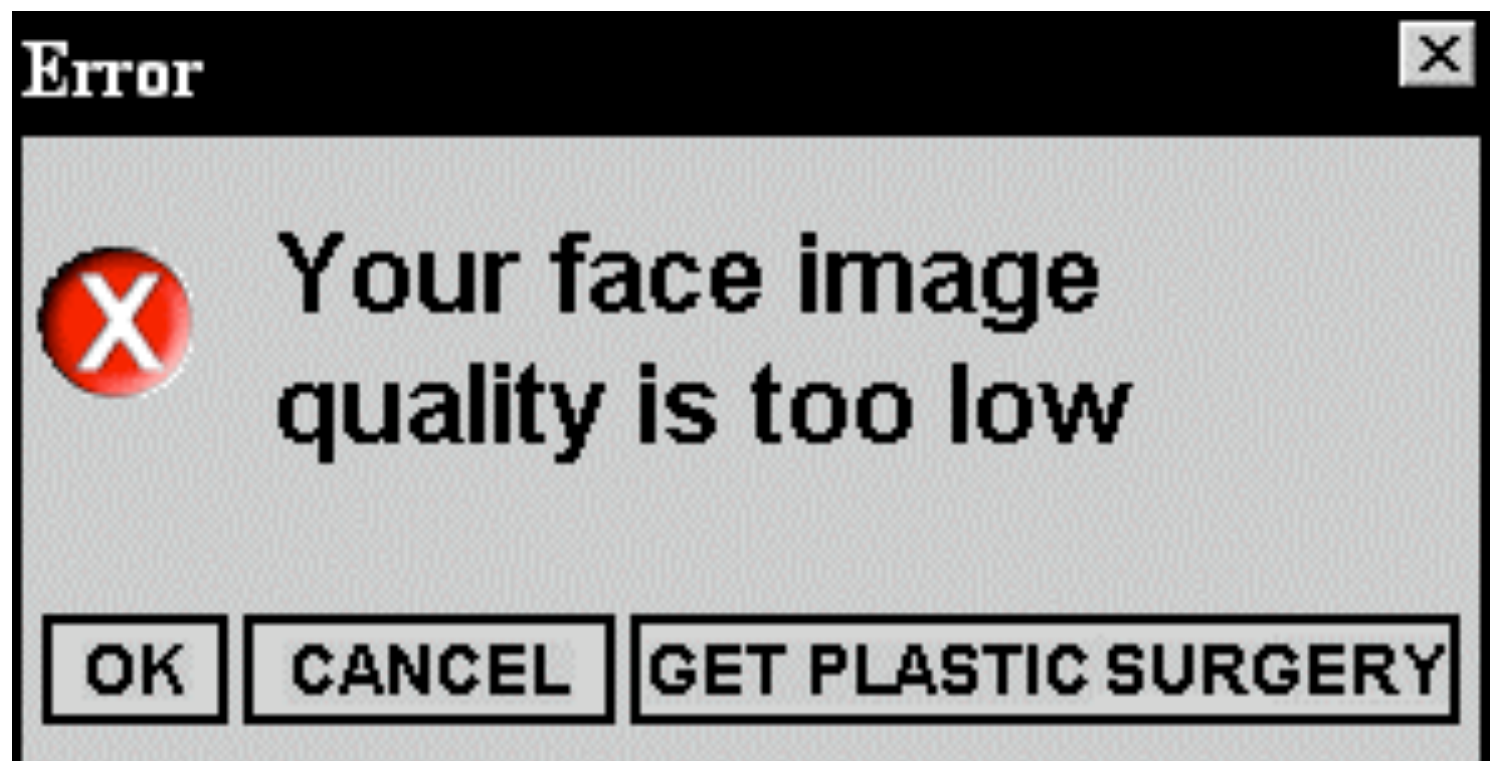

- Maybe we need another term: Clarity? 